\title{
Conservative Power Theory (CPT): A New Approach to the Tuned Passive Filter Design
}

\author{
G.J. Schäffer' ${ }^{1}$ F. A. M. $\operatorname{Moura}^{1}$ (IEEE-M) M. V. B. Mendonça ${ }^{1}$, \\ A. J. P. R. Júnior ${ }^{1}$, M. R. M. C. Albertini ${ }^{1}$ and J. R. Camacho ${ }^{2}$ (IEEE-SM) \\ Electrical Engineering Department, ${ }^{1}$ Universidade Federal do Triângulo Mineiro, Uberaba - Minas Gerais, Brazil \\ Electrical Engineering Faculty, ${ }^{2}$ Universidade Federal de Uberlândia, Uberlândia - Minas Gerais, Brazil \\ gabrielaschaffer3094@hotmail.com, fabricio.moura@uftm.edu.br.
}

\begin{abstract}
The study of the effect of the harmonic distortions and the power factor in a system with presence of nonlinear loads becomes crucial to the quality of electrical energy. Based on this problem, this article uses the Conservative Power Theory to evaluate parameters associated to product quality, aiming to obtain subsidies for the design of tuned passive filters focused on the reduction of harmonic distortion indexes associated with voltage and current, in addition to contribute to the reactive compensation of the electrical system under research.
\end{abstract}

\section{Key words}

Conservative power theory, tuned filters, power factor, harmonic distortion, power quality, power formulation theories.

\section{Introduction}

In order to increase efficiency and industrial automation, in recent years there has been a considerable growth of power electronics and microprocessor devices, among other nonlinear loads in residential, commercial, industrial and in the distribution grid. These loads generate distorted currents, that is, currents with a waveform that differs from the waveform of their power supply, with high harmonic content that when injected into the power grid, entails a variety of problems.

Thus, concerns about the quality of electric power are growing both by electric utilities and their consumers [1]. Given this scenario, the study of the quality of electric power is vital for the analysis and understanding of the electrical system, in which the commercialized product is the electric energy. In this context, there is an increase in monitoring and research around the world, for the creation of standards with reference values that can satisfy both system operators and users of the system.

Therefore, there are standards that limit the maximum levels of harmonic distortions, such as the terms proposed by the IEEE [2]. In Brazil, the limits of both harmonic distortions, as well as the consumption and reactive generation in the system are defined by ANEEL (National Agency of Electrical Energy) through module 8 of the Electric Power Distribution Procedures in the National Electric System - PRODIST [3]. The studies to meet the mentioned criteria by the standards should be carefully made and are summarized in several ways to mitigate these problems.
There are some ways to mitigate problems related to harmonic distortions, the most common of which are the use of passive harmonic shunt filters [4]. Despite the problem of introducing resonances and no follow-load changes, this technique is competitive because of the reactive power compensation and filtration efficiency combined with its low cost of deployment [5].

Currently, the use of passive filters is done according to the methodology of power theory proposed by Constantin Budeanu, criticized by the researchers of the area on its veracity. Therefore, the study of the best methodology to be used in the design of filters becomes crucial, since for the design of those, it is necessary to calculate the powers in order to obtain its reactor and capacitance.

The methodologies of power formulation must be compatible and meet the classical power theory in sinusoidal regime. In this sense, more precisely in 1927, the first theories and formulations developed by Budeanu emerge. Nevertheless, in 1932, Fryze published his theories and since then there is still no consensus on the best topology to be applied [6]. Aiming to establish a standard to be adopted by the international scientific community, the document of the Institute of Electrical and Electronics Engineers - IEEE 1459 was designed with the basic premise of the decomposition of the apparent power in fundamental and not fundamental, showing the power arising from harmonic distortions of current and voltage $[6,7]$.

However, the methodologies to date have some deficiencies related to the so-called non-fundamental powers. Thus, there are several studies referring to the best methodology to be used in the development of harmonic filters, since there is not yet a convergence of opinions by scholars and regulatory agencies [6].

Among these theories there is the Conservative Power Theory - TPC, proposed by Tenti and Mattavelli in 2003 and updated by Tenti, Mattavelli and Paredes in 2010. It is believed that such methodology brings important innovations in electrical system applications and it allows, in terms of the behaviours and characteristics of the circuits, the detailed interpretation of the electrical system [8].

Inserted in this conjuncture, the proposal of the Conservative Power Theory has as main characteristic the understanding of the electrical system through the decomposition of currents and powers, whose conservative definitions are based on Kirchhoff laws for 
tensions and currents, as well as in Tellegen's theorem. The authors of TPC demonstrate, by means of mathematical equations, the influence of the voltage distortions and frequency variations for the calculation of the reactive power of the network, which would only be conservative if the voltages are sinusoidal and the frequency is constant, this proposal quantifies the reactive power in the load by means of the average energy stored in the network by means of the energy storage elements (inductors and capacitors). It is noteworthy that such information is essential for electrical systems and also for the elaboration of harmonic filters, since the capacitors are the most sensitive elements of the filters due to the higher voltage levels to which they are subjected. For purposes of developing the discussions, this paper is structured as: the theory of conservative power presented in section 2 . In section 3 , the foundation on tuned filters. In section 4 , is demonstrated the test system used in this work. In section 5 the studies are presented, by means of the electric system in which the filter is installed, together with the comparisons between the proposed theories. In section 6 , the main conclusions about the problems studied are presented.

\section{Conservative Power Theory}

In this theory, all mathematical fundamentals follow the assumption of periodic performance that are applied to the analysis of electrical circuits, the terms of power and energy are defined in instantaneous and average terms. In addition, the current decomposition is made from the definitions of active power and reactive energy, taking into account voltage and current distortions. Next, the apparent, reactive and residual powers are defined. The reference used in this work is the one presented in 2010, by Paredes and Mattavelli, described in [9], and thus, a brief statement is made about it.

As said before, it is assumed that all quantities involved in the analysis are periodic in nature, thus considering $\mathrm{T}$ the period, the fundamental frequency being the inverse thereof and the angular frequency as $\omega=2 \pi \mathrm{f}$, we have the operators: the average value of a magnitude $x(t)$ and its Euclidean norm defined as $\vec{x} \mathrm{e}\|\mathrm{x}\|$. Let $\mathrm{X}$ be the effective value of $x$., and the internal product of two periodic quantities such as $\langle x, y\rangle$. Then, if the internal product of these quantities results in a value equal to zero, they are said to be orthogonal, and this orthogonality serves for sinusoidal functions displaced by 90 degrees or for harmonic components of different orders. This concept of orthogonality was demonstrated in Fryze's theory [10], a theory that greatly influenced TPC. Thus, the inequality for the internal product now proposed by CauchySchwartz, with its proof in [9], is defined as:

$$
\langle x, y\rangle \leq\|x\|\|y\|=X Y
$$

Where the quantities $\mathrm{X}$ and $\mathrm{Y}$ are RMS (Root Mean Square) components. Another important property is the impartial integral, considering $\mathrm{x}(\mathrm{t})$ with its null mean value, represented in equation (2), its integral in time and impartial will be represented in equations (3) and (4), respectively.

$$
\bar{x}=\frac{1}{T} \int_{0}^{T} x(t) d t(2)
$$

$$
\begin{aligned}
& x_{\int}(t)=\int_{0}^{t} x(t) d t(3) \\
& \hat{x}(t)=x_{\int}(t)-\bar{x}_{\int}(4)
\end{aligned}
$$

Where $\bar{x}$ is an average value and the term described as impartial disregards the mean value. According to Tellegen's Theorem, the scalar product of the terms $u_{l}$, $\hat{u}_{l}, \breve{u}_{l}$, voltage components, as well as the terms of the current, is in accordance with Kirchoff's laws. Thus, considering $u$ and $i$ as vectors of voltage and current, we have the collective instantaneous power, the collective instantaneous reactive energy and, respectively, their mean values:

$$
\begin{aligned}
& P=\bar{p}=\langle\underline{u}, \underline{i}\rangle=\frac{1}{T} \int_{0}^{T} \underline{u} \cdot \underline{i} d t=\frac{1}{T} \sum_{\mu=1}^{m} \int_{0}^{T} u_{\mu}(t) \cdot i_{\mu}(t) d t=\sum_{\mu=1}^{m} P_{\mu} \\
& W=\bar{w}=\langle\underline{\hat{u}}, \underline{i}\rangle=\frac{1}{T} \sum_{\mu=1}^{m} \int_{0}^{T} \hat{u}_{\mu}(t) \cdot i_{\mu}(t) d t=\sum_{\mu=1}^{m} W_{r \mu}
\end{aligned}
$$

$P, P_{\mu}, W$, and $W_{r \mu}$ are the mean values, in sinusoidal or non-sinusoidal condition, where $\mu$ represents the phase referred to and $m$ the number of harmonics. The equation (5) represents the active power that represents the permanent flow of energy per unit time through the network cut, and knowing that $P$ is a conservative component, it is additive on all the components of the network. Equation (6) represents the reactive energy, $W$. The oscillations of power and fluxes of currents in the network, caused by energy storage elements, must be taken into account and are represented, for sinusoidal condition, by the reactive power $Q$, equation (7). However, for non-sinusoidal situations, this theory introduces a new term called reactive energy, since the concept of reactive power has not yet been defined.

$$
Q=\omega W_{r}=V I \sin \varphi(7)
$$

Thus, the conservative term given for both active power and reactive energy means that energy is conservative, or rather, the energy entering a given circuit is the same as that which comes out of it. In addition, this theory makes use of active and reactive current decompositions, but with an additional component, the residual or zero current. The active current is defined as the minimum required to provide active power and is expressed by equation (8):

$$
i_{a}=\frac{\langle v, i\rangle}{\|v\|^{2}} v=\frac{P}{V^{2}} v
$$

The reactive current is defined as the minimum required to supply reactive energy $W_{n}$, this is related to the phase shift between voltage and current, whether caused by the presence of energy storage elements or by the presence of current-displacement in electronic circuits. Thus, it is expressed by equation (9):

$$
i_{r}=\frac{\langle\hat{v}, i\rangle}{\|\hat{v}\|^{2}} \hat{v}=\frac{W_{r}}{\hat{V}^{2}} \hat{v}
$$

The reactive current carries all reactive energy and no active power, since both the reactive part due to inductive elements and equivalent capacitance does not produce active power, and also, the reactive energy is proportional to the difference between the average total inductive energy and total capacitive average energy, that is, one 
compensates the other, so to represent the reactive energy, only the equivalent reactive part is used.

The residual current does not carry active power and neither reactive energy, this is, the internal product of $P_{v}=\left\langle v, i_{v}\right\rangle$ and $W_{r v}=\left\langle\hat{v}, i_{v}\right\rangle$ should result in zero, and it can be defined by the equation(10):

$$
i_{v}=i-i_{a}-i_{r}(10)
$$

Its interpretation is best seen through the frequency domain, Fourier series decomposition explicitly expressed in reference [9]. However, the terms belonging to the residual current can be defined as the dispersed active current $\left(i_{\text {as }}\right)$, the dispersed reactive current $\left(i_{\mathrm{rs}}\right)$ and the harmonic current generated by the load $\left(i_{\mathrm{g}}\right)$, resulting in the total current of:

$$
i=i_{a}+i_{r}+i_{a s}+i_{r s}+i_{g}(11)
$$

The dispersed denomination means that this term generates neither active nor reactive power. It is known that the effective values of voltage and current directly influence the power of the supply source, this power is characterized by the product of the effective values of voltage and current. Defined as apparent power, it can be decomposed into expressions (12) and (13).

$$
\begin{aligned}
& A^{2}=V^{2} I^{2}=V^{2} I_{a}^{2}+V^{2} I_{r}^{2}+V^{2} I_{a s}^{2}+V^{2} I_{r s}^{2}+V^{2} I_{g}^{2} \\
& A^{2}=P^{2}+Q^{2}+D_{a}^{2}+D_{r}^{2}+D_{g}^{2}=P^{2}+Q^{2}+D^{2}(13)
\end{aligned}
$$

The power factor, shown in equation (14), can also be composed, respectively, by the nonlinearity factor $\left(\lambda_{\mathrm{D}}\right)$ and the reactive factor $\left(\lambda_{\mathrm{Q}}\right)$, as shown in equations (14), (15) and (16):

$$
\begin{gathered}
\lambda=\frac{P}{\sqrt{P^{2}+Q^{2}+D^{2}}} \\
\lambda_{D}=\frac{D}{\sqrt{P^{2}+Q^{2}+D^{2}}} \\
\lambda_{Q}=\frac{Q}{\sqrt{P^{2}+Q^{2}}}
\end{gathered}
$$

Thus, it is possible, through the power factor, to evaluate the effects of non-active powers in a non-sinusoidal system.

The CPT method, which calculates the reactive power according to the stored reactive energy, already has some deficiencies, as well as the other formulations. Based on Fryze, this theory repeats mistakes, such as not explaining the physical meaning of the reactive current and reactive energy, which is not considered as a physical quantity [11].

\section{Passive harmonics filters}

The filtering process is related to the frequency spectrum and impedance curves of the different filters by rejection or selection of undesirable frequency signals. These filters are required to separate electric signals from currents by changing their amplitude and phase [4].

The so-called passive filters are composed of elements, such as: resistors, inductors and capacitors. For various combinations of these elements, there are different types of filters, which can be classified as either bypass or serial. The first is installed in parallel on the busbars as well as the capacitor banks. The second one is installed between two points of the same phase. When it comes to harmonic filters, the shunt configuration is most commonly used and can be classified into tuned and damped.

The tuned filters, used in this work, are series resonant circuits that present low resistive impedance at the resonant frequency. Those can be chosen to have more than one resonance frequency, depending on the arrangements of passive components R, L, and C. For frequencies smaller than the resonant frequency, the filter tends to be capacitive and at higher frequencies, it tends to be inductive. That is, at the fundamental frequency, $60 \mathrm{~Hz}$, will always be below the tuning frequency of the filter, so the filters behave as reactive compensators, or as commonly called, capacitor bank for power factor correction. In addition, because they have a simple construction, in comparison with the other techniques, they have low costs of construction and maintenance [12]. Using the three composite passive elements in a series circuit, we have the following input impedance:

$$
Z=R+j\left(\omega \cdot L-\frac{1}{\omega . C}\right)
$$

Where $\omega$ is the angular frequency of the power supply, and there will be a frequency at which the impedance will be purely resistive, called the series resonance situation, where the imaginary part will be zero, that is, the capacitive and inductive reactance's will be the same. Then, assuming that the resistive part $\mathrm{R}$ is disregarded, the angular frequency of resonance, $\omega_{0}$, can be determined by:

$$
\omega_{0}=\frac{1}{\sqrt{L . C}}=2 \pi f_{0}
$$

The frequency $\omega_{0}$ is a characteristic of the RLC circuit because it depends only on the components $\mathrm{L}$ and $\mathrm{C}$. This characteristic can be seen in Figure 1, in which the impedance module and the phase of the RLC circuit, respectively, are represented for a filter tuned, that is, a single-tuned resonant filter for only one frequency [12]. When observing Figure 1, it is noticed that the impedance at the resonance frequency is minimal, demonstrating that in series resonance the circuit will be purely resistive. Another fact to note is that by increasing the value of $\omega_{0}$, the impedance angles will be positive, characterizing the circuit as inductive at high frequencies, and at frequencies lower than the resonant frequency the circuit will be predominantly capacitive, which is expected, since in the industrial frequency of $60 \mathrm{~Hz}$, the circuit will act as a bank of capacitors for reactive compensation.
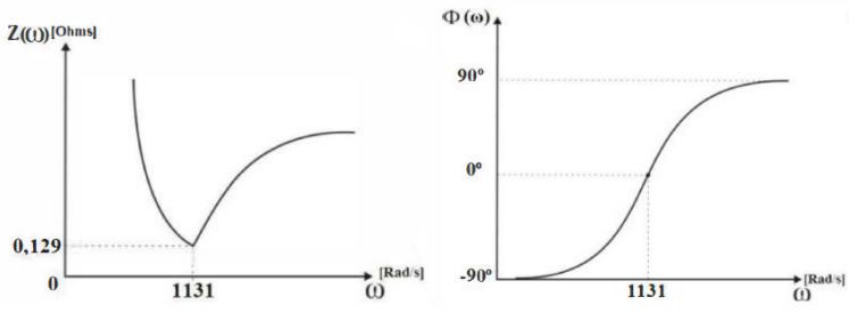

Fig.1. Filter Impedance Module and Phase.

For the base of the filter calculations it is necessary to define the quality factor $(\mathrm{Q})$, the admission factor defined by the relation between the maximum energy stored and the total energy dissipated in a period, expressed in 
equation (19). This factor determines the filter selectivity, the higher the $\mathrm{Q}$, the more selective this filter is and the higher the filter impedance will be for frequencies out of sync, and vice versa, as shown in Figure 2.

$$
Q=\frac{\omega_{o} \cdot L}{R}=\frac{1}{\omega_{0} \cdot C \cdot R}=\frac{\sqrt{\frac{L}{C}}}{R}
$$

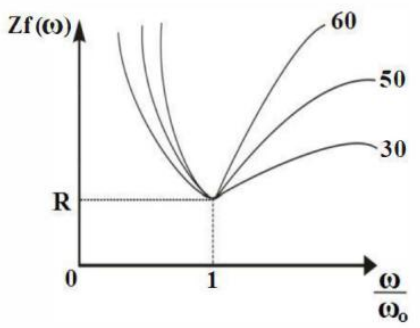

Fig.2. Impedance versus frequency for several Q factor values.

Thus, by maintaining constant resistance, as the quality factor increases, the filter impedance, at different frequencies of the resonant, will also increase. However, for the frequency of tuning, the impedance is independent of the quality factor, since it is purely resistive. Therefore, the higher the $\mathrm{Q}$ factor the more selective the filter, which prevents, substantially, the presence of harmonic currents different from the desired one flowing in the filter. However, the tuning condition is not seen in practice since in real systems the filters go through the detuning process.

\section{Electrical test system}

In order to represent the electric system in the Alternative Transients Program (ATP), we used the case study of section 6.6 Harmonic Filter Design of reference [1], whose system is represented in Fig.3.

The test system was represented by a three-phase voltage source of $13.8 \mathrm{kV}$, a delta-star transformer of 1.5 MVA, $13.8 \mathrm{kV} / 480 \mathrm{~V}$ and with $\mathrm{Xt}=6 \%$, which supplies a nonlinear load of $1200 \mathrm{kVA}$, with a power factor of 0.75 (inductive). It is also known that, without the presence of this load, there is already an individual harmonic distortion of $0.1 \%$ for the 5 th harmonic order in the transformer primary $(13.8 \mathrm{kV})$. In addition, there is the 5 th order current injection, caused by the nonlinear load rectifier, whose load represents $25 \%$ of the total industry demand [1]. The objective of this study is to design a tuned passive filter, which will be placed in parallel with the system load, with the function of filtering the 5th order harmonic present in the system. The procedures required for calculating the filter components are detailed in reference [1].

The Brazilian technical literature by ONS (System National Operator) sub-module 2.8 and module 8 of PRODIST [3], presents $6 \%$ as a reference value of individual distortions of 5th harmonic voltage, and knowing that the distortion at the low voltage busbar is $6.6 \%$, that is, a value above the allowed limit, it is necessary to implement a tuned filter. Nevertheless, in this project, a power factor correction was adopted as 0.96 and the tuning frequency in a harmonic order of 4.7, values chosen by the designer of reference [1]. Therefore, after following the calculation procedures of the components of the tuned filter, the values of inductance and capacitance were, respectively, $0.0512 \mathrm{mH}$ and $6.2165 \mathrm{mF}$.

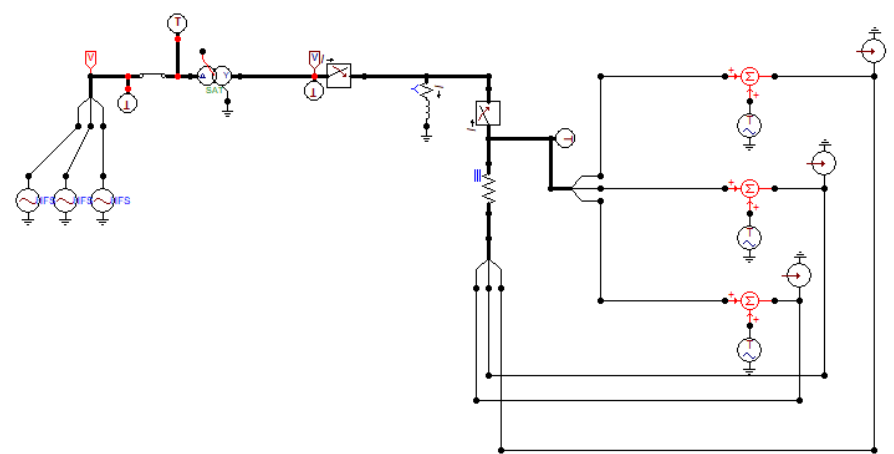

Fig.3.The electrical system in ATPDraw.

The filter design proposed by reference [1] is based on the theory of power proposed by Budeanu, and the power factor correction is done only by its value in $60 \mathrm{~Hz}$, that is, in this project, the power factor which considers the harmonics present is not corrected. In this way, the next section will present the same filter design but using the values found by the Conservative Power Theory, which calculates the power factor differently.

\section{Conservation Power Theorem - Results and discussions}

For the calculations of the power values, a power meter was first developed, based on the formulations presented in Section II, according to CPT, using the TACS (Transient Analysis of Control Systems) platform to work the equations and the decomposition of current, as well as Models (ATP programming language) for the calculation of mean values. This meter, shown in Fig. 4, is connected to the test system itself and has as input variables the voltage and the phase current.

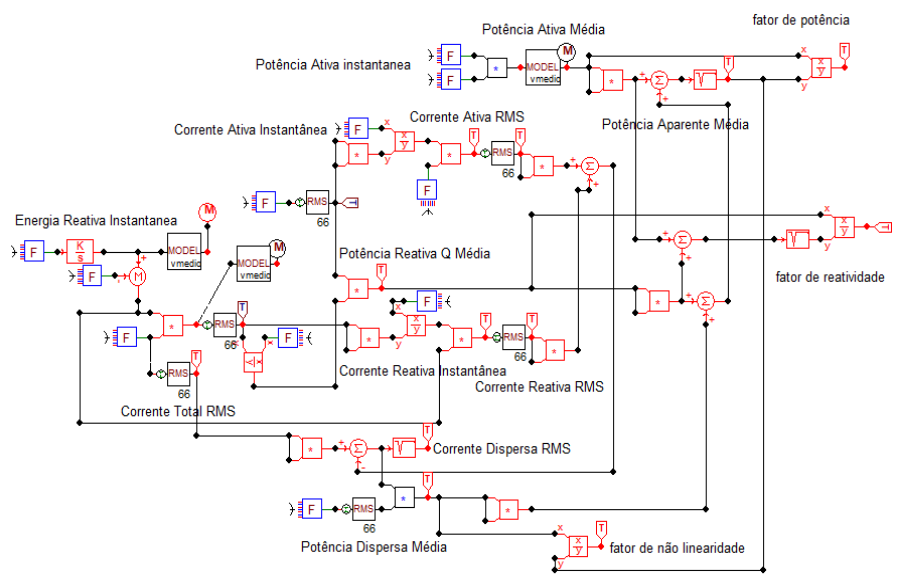

Fig. 4. CPT power meter

Thus, when the meter was connected to the system represented by Fig. 3, the following values were obtained in Table I.

\begin{tabular}{c|c|c|c}
\multicolumn{5}{c}{ Table I. Values Obtained by the CPT Meter } \\
$\boldsymbol{A}$ & $\boldsymbol{P}$ & $\boldsymbol{D}$ & $\mathbf{Q}$ \\
\hline $1.2463 \mathrm{kVA}$ & $883.42 \mathrm{~kW}$ & $290.12 \mathrm{kVA}$ & $829.86 \mathrm{kVAr}$ \\
\hline$\lambda$ & $\lambda_{D}$ & $\lambda_{Q}$ & $\boldsymbol{W r}$ \\
\hline 0.7088 & 0.2328 & 0.6846 & $2201.3 \mathrm{~J}$
\end{tabular}


The above represented values were measured on the high voltage side of the system without the addition of the filter. The problem of the power factor, 0.71 delayed, is well known, which is below the 0.92 limit established by ANEEL (Brazilian Electrical Energy National Agency)[13]. Therefore, there is a need not only to filter the 5th order harmonic but also to correct the power factor to be within the established standards. In addition to the high value of the reactive power $Q$, it can be seen that the residual power $D$ has a high value, which shows the high distortion value added to the system.

For comparison, the values, per phase, of the voltage on the low voltage side, the total current and the harmonic current drained by the load are respectively $274.46 \mathrm{~V}$, 1,463A, and 27.3A. Thus, using the same filter design procedure of reference [1], but altering the values for the measured ones, according to Conservative Power Theory, a series tuned filter was designed that was allocated in parallel with the load, whose inductance and capacitance values are respectively $0.0513 \mathrm{mH}$ and $6.217 \mathrm{mF}$. The adopted capacitor is $550 \mathrm{kVAr}$. It is noticed that the values of $\mathrm{L}$ and $\mathrm{C}$ are similar to those obtained when using the Budeanu method, but for a system whose distortion is greater, these values will be slightly different since, in the filter design, Budeanu uses the power factor at $60 \mathrm{~Hz}$, that is, values of only the fundamental frequency, ignoring the other orders present in the system and its method of powers calculating has great problems [14]. In this way, the new values after installation of the tuned filter in question are presented in Table II.

\begin{tabular}{c|c|c|c}
\multicolumn{4}{c}{ Table II. Values Obtained by the CPT Meter } \\
$\boldsymbol{A}$ & $\boldsymbol{P}$ & $\boldsymbol{D}$ & $\mathbf{Q}$ \\
\hline $962.74 \mathrm{kVA}$ & $924.06 \mathrm{~kW}$ & $39.576 \mathrm{kVA}$ & $267.23 \mathrm{kVAr}$ \\
\hline$\lambda$ & $\lambda_{D}$ & $\lambda_{Q}$ & $\boldsymbol{W r}$ \\
\hline 0.9598 & 0.0411 & 0.2778 & $708.94 \mathrm{~J}$
\end{tabular}

When observing the values obtained in Table II and comparing with Table I, the first point to be highlighted was the increase of the power factor, from 0.71 to 0.96 inductive, which was projected above the limit desirable by ANEEL. This is due to the insertion of the capacitor of the filter that acts as a capacitor bank for correction of power factor for fundamental frequency, below the tuning point and therefore, observing the equation (16), we can notice the decrease of the reactivity factor, dependent on the reactive power $Q$, which has been corrected by this compensation. Another point to emphasize is the large decrease in the residual power $D$ and, consequently, the nonlinearity factor $\lambda_{D}$, this can be better explained by observing equations (10) and (12), which show that $D$ is proportional to residual current, that is, this indicator strongly depends on the presence of non-linearity between the voltage and current waves. Thus, with the filtering of the 5th harmonic current through the filter, the residual current decreases.
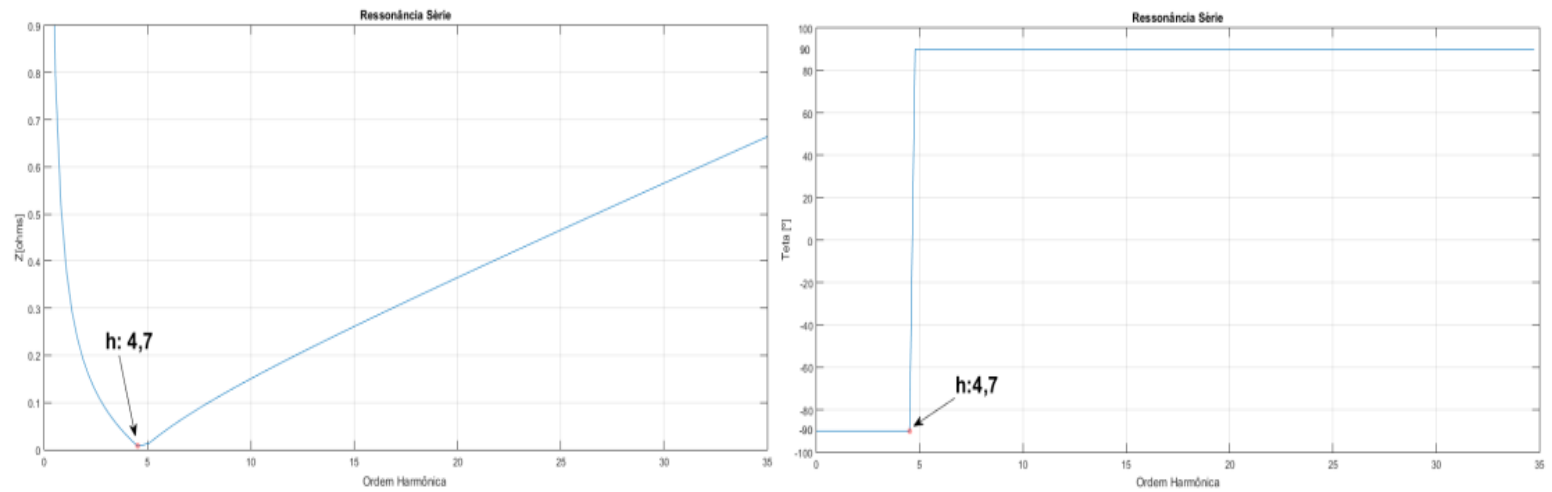

Fig. 5. Module and phase of equivalent filter impedance

The bus voltage, the total current, and the 5th order harmonic current, per phase on the low voltage side were measured again after the filter was installed. The values obtained were: $280.3 \mathrm{~V}, 1128.2 \mathrm{~A}$ and $5.32 \mathrm{~A}$. It is noticed that the voltage in the bus had a small increase due to the installed capacitor, the harmonic current decreased, as expected, and consequently decreased slightly the total RMS current. For a better example, the impedance curves of the filter and the system after the insertion of the filter are shown in figures 5 and 6 , respectively, performed in Matlab software. These curves serve to evaluate in what frequency the system will be in parallel resonance and in series resonance, if desired only for the 5th harmonic.

When looking at the filter impedance module, figure 5 shows that the filter is tuned to the expected order, there was a series resonance at 4.7, and the quality of the filter is low, as detailed in section III. Observing the phase of that figure, it can be noted that for frequencies below the tuning frequency, the filter has a capacitive behaviour and for frequencies above this, it is inductive, as expected. Then, in Figure 6, there will be a series resonance, low impedance, at 4.7, as it was designed and shown in Figure 5, and will have a high impedance parallel resonance in the 4 th order.

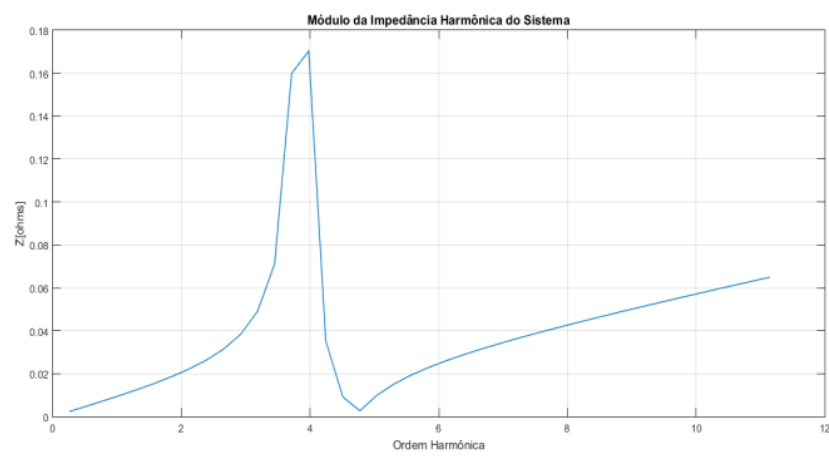

Fig.6. System Harmonic Impedance Module 
Knowing that in this system there is no presence of even harmonics, the filter can be installed safely in the observed busbar.

\section{Conclusion}

In view of the results of this work, it was possible to analyze through computer simulations, using the ATPDraw software, the power factor correction, and the mitigation of harmonic distortion, of a nonlinear load, through the use of a tuned filter. However, it was verified also the importance of the present harmonic distortion analysis for the design of a more efficient filter. Thus, the use of a power theory that best represents the physical phenomena and provides reliable parameters becomes crucial for the design of filters. It can be said that the efficiency of the filter was relevant to remove the harmonic order to which it was tuned, reaching its main objective.

Thus, although with the aim of a unified approach to power theories, which can be designed in energy metering and charging as well in energy compensation, an error-free theory has not yet been found. Therefore, knowledge of harmonic disturbances and their forms of mitigation require special attention to ensure safe and precise designs, thus avoiding the risk of the filter malfunction or the occurrence of undesirable situations such as its destruction, causing risks to the system electric equipment.

\section{References}

[1] DUGAN, R. C., Mc GRANAGHAN, M. F., Electrical power system quality. Second edition. Publisher McGraw-Hill, United States of America, 2002

[2] IEEE Standard 519-1992. IEEE Recommended practices and requirements for harmonic control in electrical power systems, 12 abril 1993.

[3] Agência Nacional de Energia Elétrica- ANEEL. Procedimentos de distribuição de energia elétrica no sistema elétrico nacional- PRODIST, Módulo 8. Disponível em: http://www.aneel.gov.br/modulo-8. Acesso em 01 de janeiro de 2017.

[4] NASCIMENTO, R.P. Proposta de Procedimentos para projetar filtros harmônicos a partir de um programa de penetração harmônica, incluindo cálculos de desempenho e suportabilidade. Dissertação de mestrado - Universidade Federal de Uberlândia, 2007.

[5] MAZA-ORTEGA, J. M. et all." A Software-based Tool for Optimal Design of Passive Tuned Filters". IEEE International Symposium on Industrial Electronics, pp. 3273-3278, Julho, 2010.

[6] LIMA, Ezequiel J. Reflexões sobre as teorias de potência elétrica em regime não senoidal. Dissertação de mestrado Universidade Federal de Uberlândia, 2014.

[7] MODESTO, S. Avaliação Computacional de Métodos de Obtenção da Potência Elétrica para Tensões e Correntes Equilibradas e Desequilibradas com Distorções Harmônicas. Trabalho de Conclusão de Curso. Engenharia Elétrica. Universidade Federal do Triângulo Mineiro, Uberaba, 2015.

[8] FRAISSAT, F.B. Study of Conservative Power Theory for Analysis of Electrical Systems.. MSc Dissertation -Universidade Estadual Paulista. Bauru, 2011. \{In Portuguese\}.

[9] PAREDES, H. K. M. Teoria da Potência Conservativa: Uma nova Abordagem para o Controle Cooperativo de Condicionadores de Energia e Considerações sobre Atribuição de Responsabilidades. Tese. Faculdade de Engenharia Elétrica e de Computação, Universidade Estadual de Campinas, 2011.
[10] DECKMANN, S.M.; POMILIO, J. A. Condicionamento de Energia Elétrica e Dispositivos FACTS. LCEE-DSCEFEEC-UNICAMP, Campinas,2009.

[11] CZARNECKI, L.S. What is wrong with the Conservative Power Theory (CPT). IEEE Transactions on Instrumentation and Measurement, 2016.

[12] VASCONSELLOS, A. B. et al. Applications of Tuned Passive Harmonic Filters in a Busbar With Non-linear Loads. IEEE Transactions on Instrumentation and Measurement, 2012.

[13] ANEEL National Electric Energy Agency (2013). Normative Resolution $\mathrm{n}^{\circ}$ 482. Accessed in June 10, 2017, in http://www.aneel.gov.br/area.cfm?idArea=757\&idPerfil=2. \{ In Portuguese $\}$

[14] SCHAFFER, G. J. et al. Comparative analysis methodologies formulation powers in the frequency domain. International Conference on Renewable Energies and Power Quality (ICREPQ'17), 2017. 